\title{
New insights in diagnostic thresholds for total serum bile acid (TSBA) in intrahepatic cholestasis of pregnancy (ICP)
}

\author{
Cristina Manzotti ${ }^{1}$ \\ ${ }^{1}$ University of Milan
}

April 27, 2021

\section{New insights in diagnostic thresholds for total serum bile acid (TSBA) in intrahepatic cholesta- sis of pregnancy (ICP)}

Manzotti $C$

Manzotti Cristina, MD

Department of Pathophysiology and Transplantation

University of Milan

Via Francesco Sforza 35

20122 Milan, Italy

Email: cristina.manzotti@unimi.it

Intrahepatic cholestasis of pregnancy (ICP) is a pregnancy specific liver disorder known to be associated with fetal adverse events, in particular stillbirth. At present, an effective treatment to reduce the risk of stillbirth, does not exist. Ursodeoxycholic acid (UDCA) is often given in clinical practice to reduce maternal symptom of pruritus but without strong evidence (Walker KF et al. Pharmacological interventions for treating intrahepatic cholestasis of pregnancy. Cochrane Database Syst Rev. 2020 Jul 27;7(7):CD000493), and iatrogenic preterm birth is induced when the risk of stillbirth is thought to be higher (Ovadia $\mathrm{C}$ et al. Association of adverse perinatal outcomes of intrahepatic cholestasis of pregnancy with biochemical markers: results of aggregate and individual patient data meta-analyses. Lancet. 2019 Mar 2;393(10174):899-909).

Total serum bile acid (TSBA) have been used as the most reliable marker for the diagnosis, follow-up and prognosis of ICP, but their diagnostic accuracy has been questioned (Manzotti C et al. Total serum bile acids or serum bile acid profile, or both, for the diagnosis of intrahepatic cholestasis of pregnancy. Cochrane Database of Systematic Reviews 2019, Issue 7. Art. No.: CD012546).

In this context, the paper by Mitchell A et al presents three different studies.

The first study measured fasting and non fasting TSBA levels in women with and without ICP, to assess diurnal TSBA variation. Authors found that TSBA increased markedly postprandially in both groups of women: this means that using a fasting sample, as most often occurs in clinical practice, the majority of women with severe ICP may not be diagnosed.

The second study determined TSBA non-fasting reference interval for in the third trimester of pregnancy in healthy pregnant women, by retrospective analysis of non-fasting serum samples from a local database. They found that the upper limits for normal reference interval were $10.4,15.5$ and $18.3 \mu \mathrm{mol} / \mathrm{l}$ for white, asian and black women respectively. Applying as the threshold to diagnose ICP 19umol/l, a higher limit than used in clinical practice, some affected women with mild disease would not have been diagnosed. 
However, the third study found that there was no higher risk of adverse events in this group of women, compared to a healthy pregnant population, except from iatrogenic preterm birth.

Nowadays clinical practice with pregnant women affected by ICP is still far from being evidence based, mostly because of low quality and underpowered studies published in the past, leading to heterogeneous management in antenatal clinics. Some publications of the last three years added new insights into ICP management. The paper by Mitchell A et al, even if with limits of low number of patients with ICP enrolled and unclear participant selection, seems a further contribution in this direction.

Appling post-prandial testing for pregnant women suspected to have ICP could give a more attendible sight over TSBA concentration to which foetus is exposed, improving diagnostic accuracy for ICP and understanding which pregnant women need more attention. The higher TSBA upper limit proposed of 19 $\mu \mathrm{mol} / \mathrm{L}$ may avoid overdiagnosis and over-medicalization without losing potential adverse events. New high quality and large population based clinical research is needed to reach more solid evidence. 